\title{
Use of Mueller matrix polarimetry and optical coherence tomography in the characterization of cervical collagen anisotropy
}

Joseph Chue-Sang Yuqiang Bai

Susan Stoff

Mariacarla Gonzalez

Nola Holness

Jefferson Gomes

Ranu Jung

Amir Gandjbakhche

Viktor V. Chernomordik

Jessica C. Ramella-Roman 


\title{
Use of Mueller matrix polarimetry and optical coherence tomography in the characterization of cervical collagen anisotropy
}

\author{
Joseph Chue-Sang, ${ }^{\text {a }}$ Yuqiang Bai, ${ }^{a}$ Susan Stoff, ${ }^{a}$ Mariacarla Gonzalez, ${ }^{a}$ Nola Holness, ${ }^{b}$ Jefferson Gomes, ${ }^{a}$ \\ Ranu Jung, ${ }^{a}$ Amir Gandjbakhche, ${ }^{c}$ Viktor V. Chernomordik, ${ }^{c}$ and Jessica C. Ramella-Roman ${ }^{a, d, *}$ \\ ${ }^{a}$ Florida International University, Department of Biomedical Engineering, Miami, Florida, United States \\ ${ }^{\mathrm{b}}$ Florida International University, Nicole Wertheim College of Nursing and Health Sciences, Miami, Florida, United States \\ 'Eunice Kennedy Shriver National Institute of Child Health and Human Development, Rockville, Maryland, United States \\ ${ }^{d}$ Florida International University, Herbert Wertheim College of Medicine, Miami, Florida, United States
}

\begin{abstract}
Preterm birth (PTB) presents a serious medical health concern throughout the world. There is a high incidence of PTB in both developed and developing countries ranging from $11 \%$ to $15 \%$, respectively. Recent research has shown that cervical collagen orientation and distribution changes during pregnancy may be useful in predicting PTB. Polarization imaging is an effective means to measure optical anisotropy in birefringent materials, such as the cervix's extracellular matrix. Noninvasive, full-field Mueller matrix polarimetry (MMP) imaging methodologies, and optical coherence tomography (OCT) imaging were used to assess cervical collagen content and structure in nonpregnant porcine cervices. We demonstrate that the highly ordered structure of the nonpregnant porcine cervix can be observed with MMP. Furthermore, when utilized ex vivo, OCT and MMP yield very similar results with a mean error of $3.46 \%$ between the two modalities. $\odot 2017$ Society of Photo-Optical Instrumentation Engineers (SPIE) [DOI: 10.1117/1.JBO.22.8.086010]
\end{abstract}

Keywords: anisotropy; birefringence; collagen; polarized light imaging; Mueller matrix; optical coherence tomography.

Paper 170222PR received Apr. 10, 2017; accepted for publication Jul. 24, 2017; published online Aug. $29,2017$.

\section{Introduction}

Preterm birth (PTB) has many causes, but irrespective of its etiology ${ }^{1}$, mechanical cervical failure or change in the cervix extracellular matrix (ECM) is a common endpoint. Fibrillar collagen is the major structural protein in the cervix that determines its load-bearing capabilities. With progression of pregnancy, the cervix undergoes changes in the collagen structure and corresponding mechanical strength. Structural defects in the cervix result in PTB as exemplified in women with cervical insufficiency. Several human and animal studies have suggested that atypical changes in the ECM of the cervix precede PTB. ${ }^{2-6}$ Thus, the development of diagnostic modalities that could identify premature abnormal cervical remodeling holds great potential as a tool for early and accurate assessment of cervical disease. Numerous imaging technologies are under exploration though none has yet yielded established clinical tools. ${ }^{7,8}$ Research groups have utilized second harmonic generation (SHG), a nonlinear microscopy modality, to obtain exquisite images of cervix collagen fibers (alignment, porosity, and fiber size) in the cervix of nonpregnant and pregnant women and mice, and have shown that alteration in collagen structure and assembly corresponds to the biomechanical properties of the cervix during pregnancy. ${ }^{8-11}$ The use of SHG in the clinical setting is yet hampered by the instrumentation's low field of view $(<500 \mu \mathrm{m})$ and high susceptibility to movement artifacts. At this stage, SHG systems are also expensive because of the use of femtosecond Ti:sapphire lasers.

*Address all correspondence to: Jessica C. Ramella-Roman, E-mail: jramella@ fiu.edu

\subsection{Cervix}

The cervix is an extension of the lower part of the uterus comprising a portion of the female reproductive system. It is cylinder shaped, $\sim 3$ - to $4-\mathrm{cm}$ long and $3 \mathrm{~cm}$ in diameter, and has a central canal through its entirety. A cartoon representation of the cervix is shown in Fig. 1. The cervical canal serves as the entrance, via the vagina, for sperm in reproduction and as an exit canal, via the uterus, for childbirth. The two ends of the canal are termed internal orifice (OS) connecting to the uterus and external OS at the vagina. The cervix is divided into two regions because of their cellular differences: the ectocervix and the endocervix. The ectocervix, the lower area, is visible through the vaginal opening and is comprised of a stratified squamous epithelium, having several cell layers of differing morphological characteristics. ${ }^{12}$ The thickness of the epithelial layer is between 200 and $500 \mu \mathrm{m} .{ }^{13-15}$ The dramatic structural changes undergone by the cervix during pregnancy have been categorized by four main phases: cervical softening, ripening, dilation, and repair (postpartum). ${ }^{6,16,17}$

The physiological and mechanical properties of the cervix are altered by a cascade of microenvironmental events in each of these phases, which are not completely understood. Cervical softening begins within 1 month of the initiation of pregnancy and the cervix undergoes increased vascularity and edema. ${ }^{6}$ Softening is a longer phase than the others, progressing through the 33rd week of pregnancy. During this phase, the collagen of the stroma becomes less organized and the cervix becomes pliable and begins shortening. ${ }^{17}$ The ripening phase

1083-3668/2017/\$25.00 @ 2017 SPIE 


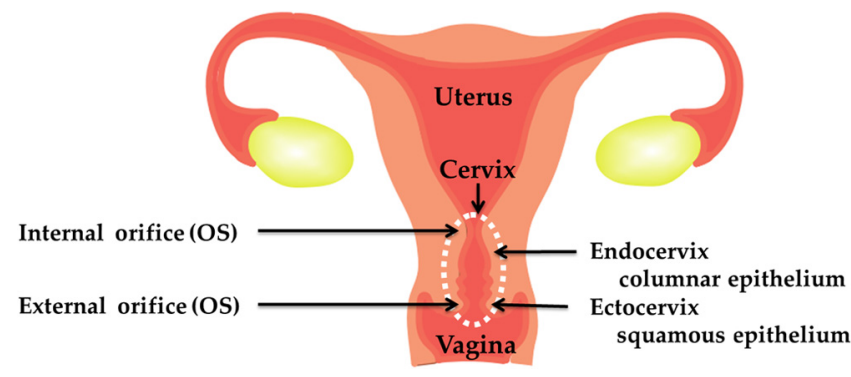

Fig. 1 The cervix.

begins after softening, in this phase the cervix goes from a loadbearing function to a birthing canal. ${ }^{18}$ By the end of cervical ripening, when dilation begins, a $30 \%$ to $70 \%$ decrease of collagen $^{16,19,20}$ occurs since the onset of pregnancy. Others have shown an increased collagen solubility in weak acids rather than change in collagen content, ${ }^{21,22}$ and an increase in water $(5 \% \text { to } 10 \%)^{18,23,24}$ with a shift from insoluble to soluble collagen ${ }^{19,25}$ with as much as $90 \%$ soluble collagen by the third trimester. ${ }^{18}$ Dilation follows ripening and involves an influx of leukocytes, ${ }^{18}$ similar to an inflammatory response, which may serve to cause an increase in the matrix metalloproteinase, collagenase. ${ }^{6,26,27}$ Collagenase causes the breakdown of collagen cross links and allows the cervix to weaken ${ }^{21,28,29}$ and open, thereby radically changing shape by shortening and effacing in preparation for delivery of the baby.

\subsection{Collagen in the Cervix}

An ECM lies under the epithelium of the cervix, separated by the thin basal lamina layer of collagen type IV fibers, ${ }^{13}$ and consists mainly of collagen, $\sim 10 \%$ to $15 \%$ smooth muscle cells $^{18,30,31}$ and a ground mixture of biomolecules.

Two types of collagen comprise the cervical ECM; 70\% is collagen type I and $30 \%$ is collagen type III.

Numerous researchers have studied the collagen of the uterus to determine how this structure maintains its integrity during pregnancy. ${ }^{6,10,28,32-36}$ Aspden found the structure of collagen is oriented in three unique areas surrounding the cervical canal, the anisotropic alignment of the collagen differing within each area. The cervical fibrils are aligned both around and along the canal for increased strength as shown in Fig. 2..$^{21,37-39}$

In previous work, Gan et al. ${ }^{40}$ utilized unpolarized spectral domain optical coherence tomography (OCT) to determine the distribution of collagen in the cervix. Their algorithm uses preprocessing of images to first promote edge detection of fibers in different depth planes of the OCT acquired threedimensional stack before determining the orientation of subsections in the planes. This is done over selected regions in the sample. The orientation information across different regions can then be recomposed to create a complete orientation map of the entire region that was imaged.

We proposed the use of an optical modality, Mueller matrix polarimetry (MMP), to target the fibrous ultrastructure organization of the cervix. This paper describes how MMP can be used to ascertain collagen arrangement in the cervix and will validate its findings with OCT.

\section{Materials and Methods}

A combined OCT-MMP system introduced in previous work ${ }^{41}$ was modified to a fiber-based OCT system shown in Fig. 3. The system is based on a Michelson interferometer. The laser light source is a broadband superluminescent diode (Bayspec, San Jose, California) with 840-nm central wavelength and 50-nm full width at the half-maximum bandwidth. A telecentric scan lens (LSM03-BB, Thorlabs, Newton, New Jersey) focuses the light onto the sample and collects the backscattered light. Galvanometer-mounted mirrors (GVS012, Thorlabs, Newton, New Jersey) on the sample arm enable transverse beam scanning on the sample. A custom-made spectrometer detects and measures the interference signal between reference arm and sample. The spectrometer consists of a collimating lens with a focal length of $75 \mathrm{~mm}$ (Edmund Optics, Barrington, New Jersey), an 1800 lines $/ \mathrm{mm}$ volume holography transmission grating (Wasatch Photonics, Logan, Utah), an assembly of triple lenses with an effective focal length of $150 \mathrm{~mm}$, and a line array CCD camera (spL4096-140k, Basler, Highland, Illinois). Data processing algorithm, control and display software are developed using MATLAB (Mathworks, Natick, Massachusetts). The images produced by the system have a theoretical resolution in depth of $5 \mu \mathrm{m} /$ pixel, and an axial resolution of $20 \mu \mathrm{m} /$ pixel.

The coregistered Mueller matrix system shown in Fig. 3 is traced by the dashed green line and consists of a CCD camera (Lu175, Lumenera, Ottawa, Ontario) with a Computar MLH$10 \times 1 / 2$ in 13 to $130 \mathrm{~mm}$ f5.6 $10 \times$ macrozoom lens. The camera was secured above the sample objective to allow focusing on the height adjustable stage. A linear polarizer (Prinz, Northbrook, Illinois) and two liquid crystal retarders (LCR) (Meadowlark Optics, Frederick, Colorado) between the lens and sample objective form the polarization state analyzer of the polarimeter. Employing the same sample objective for the

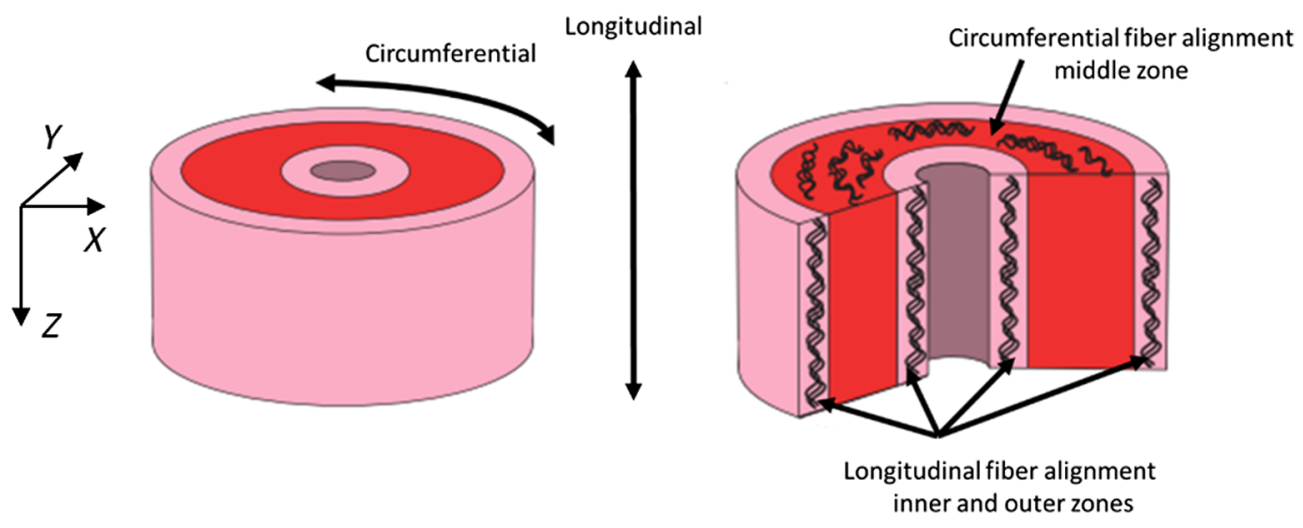

Fig. 2 Alignment of collagen fibers in human cervix in three anisotropic zones. 


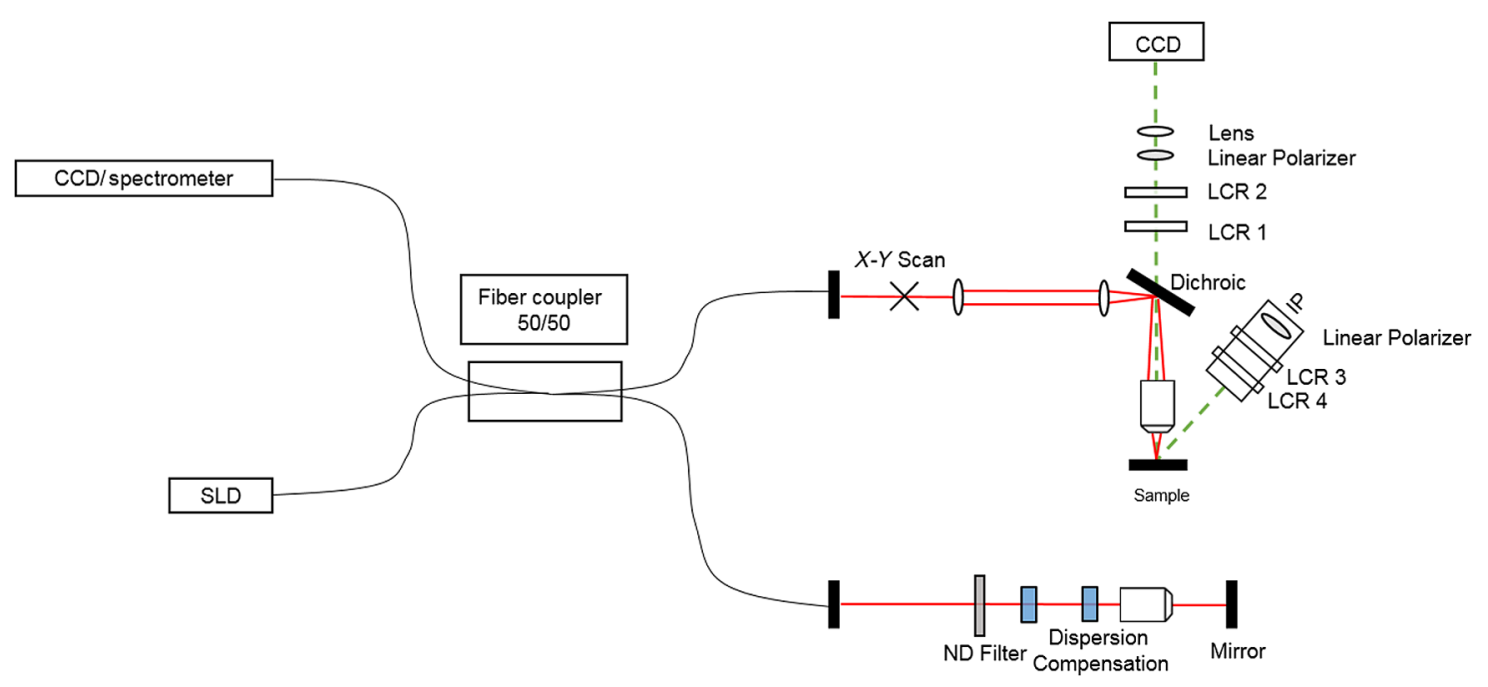

Fig. 3 Combined OCT and Mueller matrix system schematic (red light, OCT; green dashed, MMP).

two imaging systems ensures they are imaging the same region of interest. The illumination port in Fig. 3 indicates the light source for the MMP. A $530 \mathrm{~nm}$ LED (M530L, Thorlabs, Newton, New Jersey) was oriented to illuminate the sample at an incident angle of $45 \mathrm{deg}$ and collimated with a $30-\mathrm{mm}$ diameter tube and a 75-mm focal length plano-convex lens (Newport, Irvine, California). Two LCRs were mounted after the light source and a linear polarizer using a cage system to form the polarization state generator. The calibration of the MMP system follows a standard methodology utilized by our group in several applications ${ }^{42,43}$ and resulted in a condition number of 2. A Mueller matrix of air was also acquired and showed an error well below $1 \%$.

\subsection{Mueller Matrix Decomposition}

Mueller matrix imaging is a useful polarimetry technique as the $4 \times 4$ Mueller matrix completely characterizes the polarimetric properties of a sample. ${ }^{44,45}$ The decomposition of the Mueller matrix (M) (as proposed by Lu-Chipman ${ }^{46}$ ) yields three canonical matrices $\mathbf{M}=\mathbf{M}_{\Delta} \mathbf{M}_{R} \mathbf{M}_{\mathrm{D}}$ : a $\mathbf{M}_{\Delta}$ accounting for the depolarizing effects of the material, a retarder matrix $\mathbf{M}_{R}$ for the effects of the material linear birefringence and optical activity, and a diattenuator matrix $\mathbf{M}_{\mathrm{D}}$ includes the effects of linear and circular diattenuation. ${ }^{46-49}$ The resulting matrices can be analyzed to yield quantitative medium properties that have a demonstrated $^{50}$ useful diagnostic power and are used in this study. We have identified two such parameters as relevant to this study: linear retardance $\delta$, birefringence which relates to the abundance of collagen in the cervix and slow axis orientation $\theta$, which is directly related to the orientation of the collagenous structures in the tissue. The orientation calculation used was derived by Ma et al. ${ }^{51}$ Another parameter that may be of interest is depolarization, with which we can observe changes in the ECM, such as shortening and thickening of collagen. Because the information decomposed from a Mueller matrix is contained in each pixel of the image, no processing is required afterward to generate parameters of interest. As an example, retardation axis orientation is directly calculated from the Mueller matrix at each pixel to generate the orientation images. The MMP images show a resolution of $12.0 \mu \mathrm{m} /$ pixel.
Circular statistics ${ }^{52,53}$ is a subset of statistics for data that can be shown on a unit circle such as directions where the sign of values is determined by the direction of rotation. The periodicity of such data requires a departure from normal arithmetic statistics, which would give a faulty representation of the mean of the data set. Circular statistics was applied to the orientation data decomposed from the Mueller matrix to calculate directional parameters. This method requires that the data first be transformed into unit vectors with two-dimensional data as shown in Eq. (1). Equation (2) is the mean resultant vector $\bar{r}$ of the data set. The mean angular direction $\bar{\alpha}$ can be calculated using the four quadrants inverse tangent of $\bar{r}$.

$r_{i}=\left(\frac{\cos \alpha_{i}}{\sin \alpha_{i}}\right)$,

$\bar{r}=\frac{1}{N} \sum_{i} r_{i}$,

Kurtosis and mean angle were calculated in our study. Circular kurtosis is the measurement of outliers in a distribution, and is associated with the weight of the tails in a data set. ${ }^{54}$ It is useful as a measurement of how uniform a distribution of angles is in a data set, which can be confounded in mean calculations where a wide array of values can equal a certain mean angle depending on their frequency. An equal distribution of angles will give a kurtosis of 0 , whereas a narrow distribution of angles will move toward 1.

\subsection{Optical Coherence Tomography Image Processing}

We aim to mimic Gan et al.'s approach to measure the orientation of birefringent tissue using OCT, and to compare it with MMP, which should generate orientation information inherently summed across all depths in the sample for the entire field of view. Our approach preprocessed and averaged the en face data in a similar manner suggested by Gan et al. The determination of orientation, however, was calculated using our own method, which applies the Radon transform and calculation of the projection angle SNR as shown in Fig. 4. The Radon 


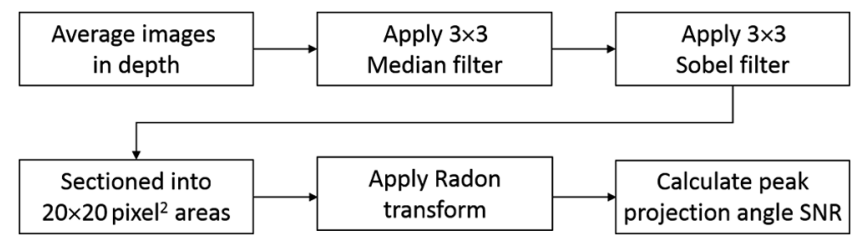

Fig. 4 OCT processing pipeline.

transform projects the sum of image intensities along the different orientation angles inputted into the transformation. ${ }^{55}$

Five $X-Y$ images (c-scans) were first averaged in the $Z$-direction to improve the orientation measurements across a depth of $40 \mu \mathrm{m}$. The c-scans were then smoothed with a $3 \times 3$ median filter to remove speckle noise. The resultant matrix was then convolved with a $3 \times 3$ Sobel filter to generate an image with emphasized structural edges. This matrix was sectioned into $20 \times 20$ pixel areas and Radon transformed. The SNR of all projection angles was calculated for the Radon transformed section using Eq. (3) to produce a graph, such as in Fig. 5. The SNR is defined here as the quotient of the standard deviation of a projected angle with the standard deviation of the entire image section. The peak projection angle SNR is denoted by a triangle and selected to be overlaid over the section of the enface image of the sample as a line. This peak corresponds to the orientation of the sample as it is calculated for sections throughout an enface image of the sample. ${ }^{55}$

$\mathrm{SNR}=\frac{\sigma_{R}(\theta)}{\sigma_{1}}$.

The orientations measured by both imaging techniques were superimposed over the images they were calculated from as oriented lines. OCT was used as a standard for testing the effectiveness of MMP in this application.

\subsection{Anisotropic Test Samples}

Two test samples were used to verify the efficacy of the OCTMMP's orientation measurements. The first sample is an

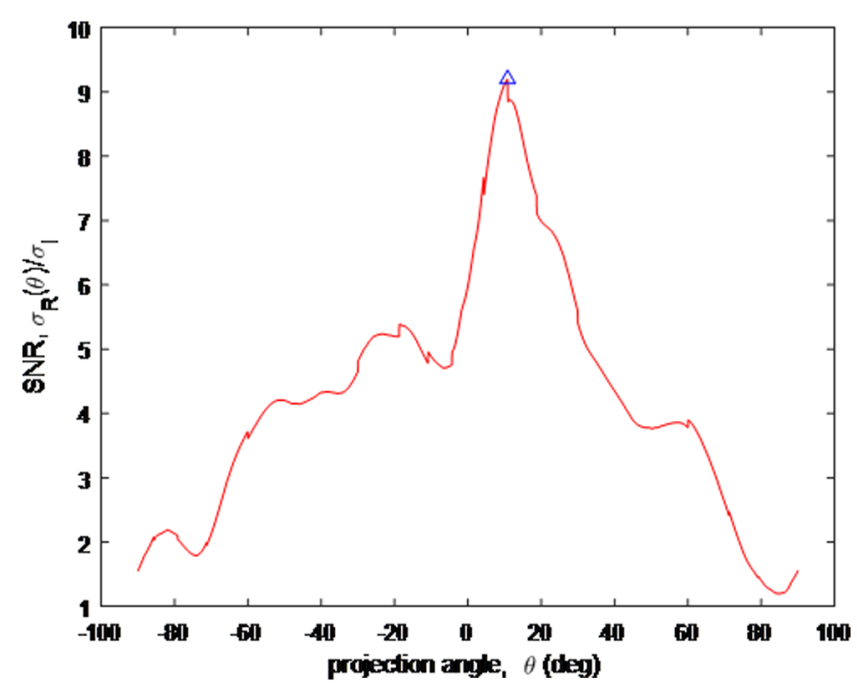

Fig. 5 Radon transform of section of silicon phantom. extruded silicon phantom. The extrusion process creates striations oriented in a single direction, which are easily observed by eye and measured by a protractor creating a highly anisotropic sample with a known retardation axis. Its low scattering and absorbing properties as a mostly transparent material ensured that there would be minimum loss of polarization in the light returning to the MMP. It produced the best measure of orientation out of all the samples used. Porcine tendon was another test sample with high anisotropy that was investigated. Highly ordered collagen in tendon has a strong and uniform birefringence, which is the basis for the MMP's calculation of retardance and orientation. Both orientation samples were rotated on the OCT-MMP sample stage and then imaged.

\subsection{Cervical Samples}

Fresh porcine cervices were obtained from the abattoir ranging from 1 to $2 \mathrm{~cm}$ in diameter. They were fixed in $4 \%$ paraformaldehyde and embedded in paraffin for preservation. The embedding process first began with dehydrating the tissue with successive washes of ethanol $(\mathrm{EtOH})$ from $50 \%$ to $100 \%$ concentration in steps of $10 \%$ for 10 min each wash. The washing was repeated with solutions of EtOH:Citrisolve in concentrations of $2: 1,1: 1$, and $1: 2$, and then three washes of $100 \%$ Citrisolve. Last, Citrisolve was exchanged for paraffin in a vacuum oven set between $54^{\circ} \mathrm{C}$ and $58^{\circ} \mathrm{C}$. First washes of $2: 1,1: 1$, and 1:2 Citrisolve:paraffin were done for $30 \mathrm{~min}$ each before using $100 \%$ paraffin for 1 to $2 \mathrm{~h}$ and then leaving it overnight in $100 \%$ paraffin. The cervices were then put in place before the paraffin was set to hard. A total of 10 cervices were imaged; the images reported are representative of the samples.

\section{Results and Discussion}

All images shown are of the $Y-X$ plane of the cervix denoted in Fig. 2. Orientation of 0 deg is parallel to the $X$ axis and a positive $\Delta \theta$ is considered counterclockwise from horizontal. The silicon phantom was measured first. Its weak attenuation can be seen in the low depolarization value of the phantom seen in Fig. 6(b), the uniform value of retardation in Fig. 6(c). The dashed line box in Fig. 6(a) is the region where orientation was compared between OCT and MMP in Fig. 7.

The circular statistics shown in Figs. 7(b) and 7(d) was calculated from the regions encased by the dashed line squares in Figs. 7(a) and 7(c). It is important to note that unlike the MMP images that are taken at the surface of the sample, the OCT images are taken below the surface of the sample as they are c-scans averaged in depth. This is why striations can be seen by both imaging modalities in the silicon phantom which was completely uniform in structure throughout the sample but not in the tissue samples. Both imaging modalities had kurtosis values in the 90-percentile range indicating a tight distribution of angles around a mean angle of $68 \mathrm{deg}$ and $65 \mathrm{deg}$ for the OCT and MMP, respectively. After the orientation measurements were confirmed to properly change as the phantom was rotated on the sample stage, we moved to tendon as a biological sample which would attenuate light due to scattering and absorbing more than the silicon phantom but still have high anisotropy which would be seen in the orientation measurements.

The greater attenuation of the tendon is evidenced by the much greater depolarization value in Fig. 8(b) as compared to the silicon phantom. Despite its high anisotropy, tendon does not exhibit retardation as uniform as the silicon phantom. The changes in morphology and roughness of the tendon's 

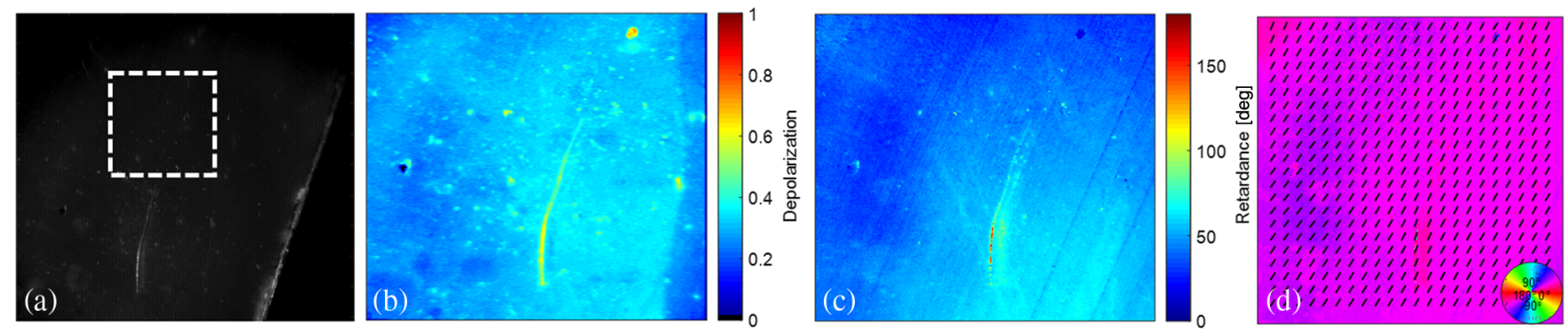

Fig. 6 Silicon phantom Mueller matrix decomposition: (a) CCD image, (b) Mueller matrix decomposed depolarization, (c) Mueller matrix decomposed retardation, and (d) Mueller matrix decomposed orientation.
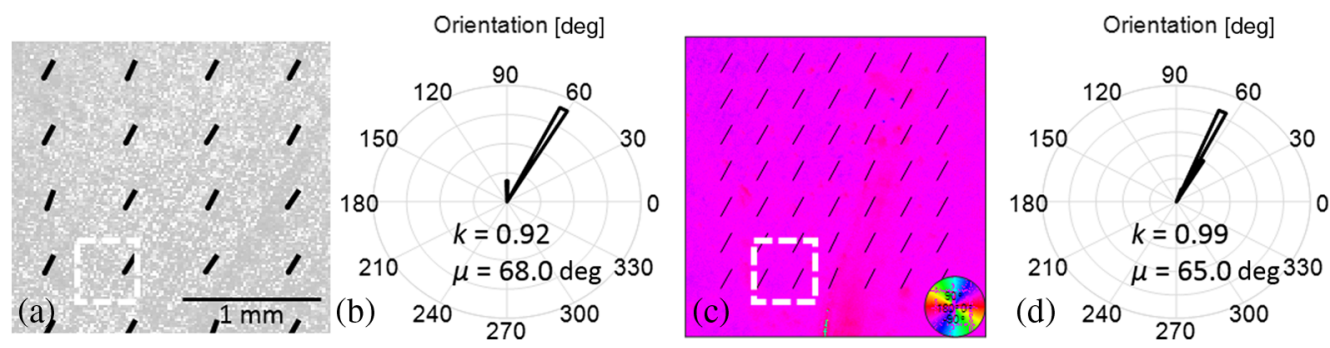

Fig. 7 Silicon phantom OCT-MMP orientation comparison: (a) OCT c-scan, (c) Mueller matrix decomposed orientation, and (b/d) orientation histogram circular statistics ( $k=$ kurtosis, $\mu=$ mean angle).
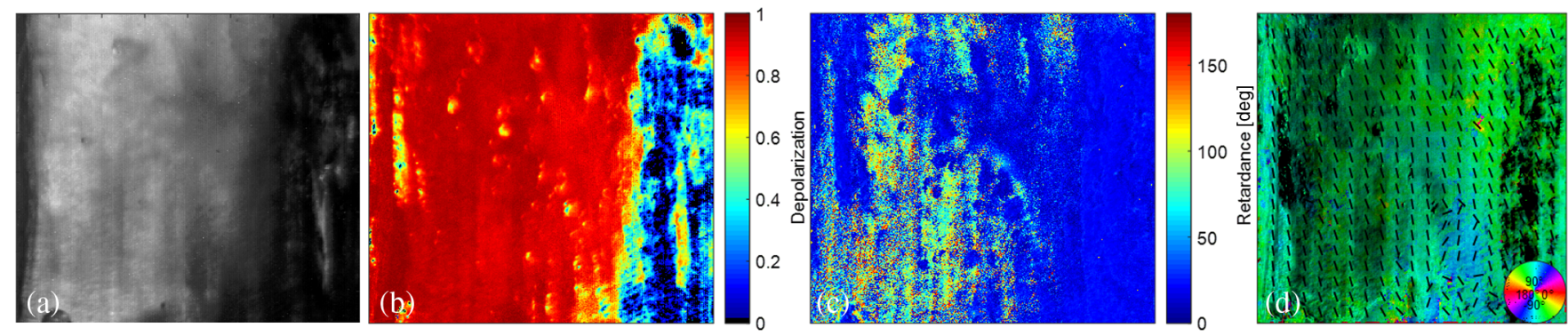

Fig. 8 Tendon Mueller matrix decomposition: (a) CCD image, (b) Mueller matrix decomposed depolarization, (c) Mueller matrix decomposed retardation, and (d) Mueller matrix decomposed orientation.

superficial layers compared to the silicon phantom can be seen as deviations in the orientations calculated by OCT and MMP in Figs. 9(a) and 9(c).

A breakdown in the Mueller matrix orientation is highlighted by a phase wrapping of orientation angle from +70 deg to -70 deg. Regardless, the tendon produced high kurtosis values with both imaging modalities around a mean angle of $-74 \mathrm{deg}$ in the selected area.
Once both imaging techniques had been tested on the anisotropic test samples, we imaged the paraffin embedded cervices to see whether the circumferential alignment of cervical collagen, which is a birefringent material, could be resolved by the techniques. The inner and outer regions of the cervix contain collagen oriented in the $z$-direction, which cannot be resolved with polarimetry of the sample's surface. We are interested in the region between the OS and the outer edges of the cervix
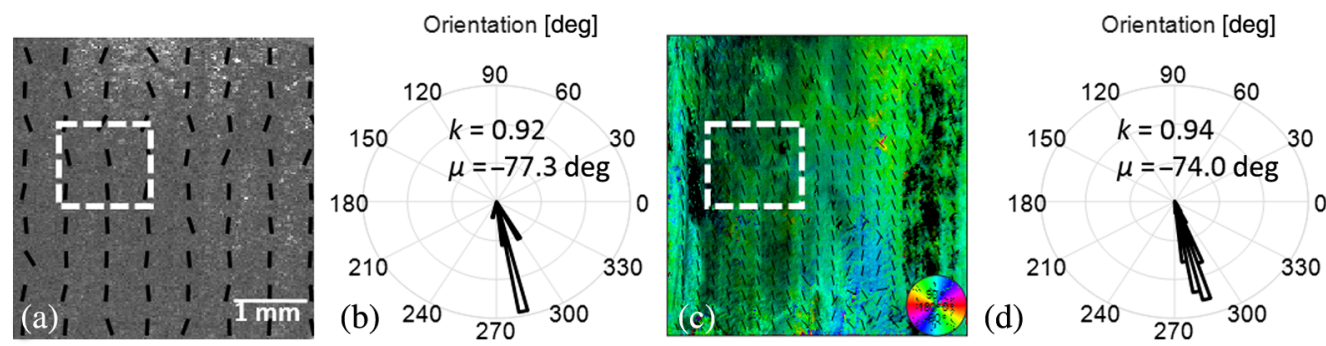

Fig. 9 Tendon OCT-MMP orientation comparison: (a) OCT c-scan, (c) Mueller matrix decomposed orientation, and (b/d) orientation histogram circular statistics ( $k=$ kurtosis, $\mu=$ mean angle). 

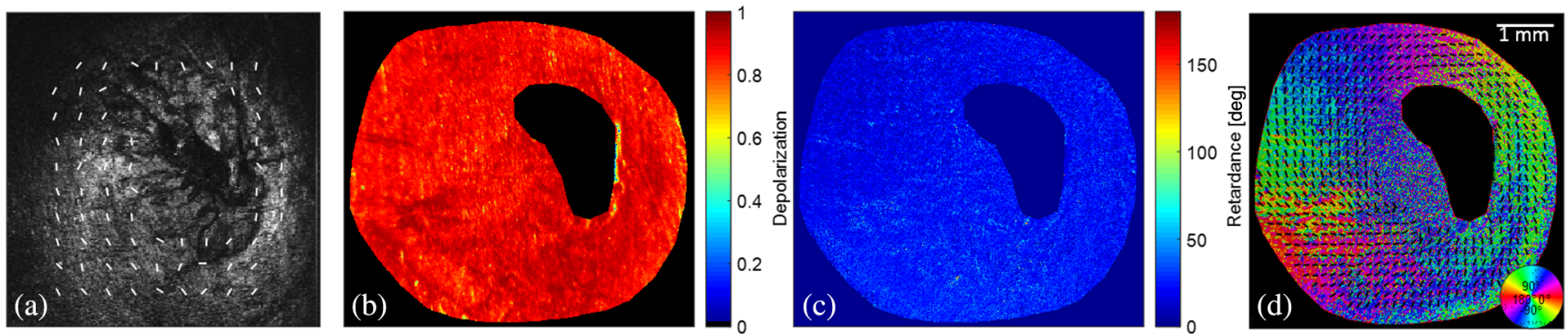

Fig. 10 Paraffin embedded cervix 1 Mueller matrix decomposition: (a) CCD image, (b) Mueller matrix decomposed depolarization, (c) Mueller matrix decomposed retardation, and (d) Mueller matrix decomposed orientation.
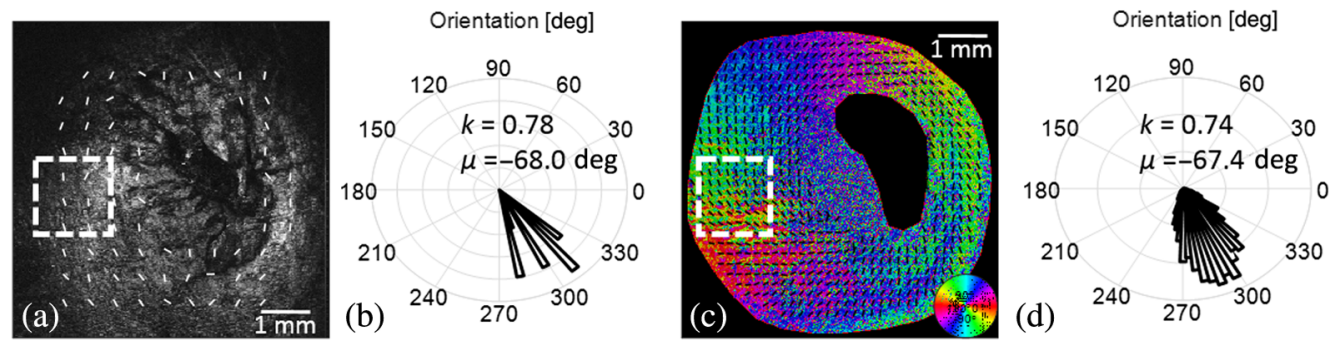

Fig. 11 Paraffin embedded cervix 1 OCT-MMP orientation comparison: (a) OCT c-scan, (c) Mueller matrix decomposed orientation, and (b/d) orientation histogram circular statistics ( $k=$ kurtosis, $\mu=$ mean angle).

because it contains collagen circumferentially aligned around the OS and can be resolved noninvasively with polarimetry of the surface. Two different cervices are shown using OCT in Figs. 11(a) and 13(a). ${ }^{40}$ Similarly, the orientations calculated by MMP also rotate around the OS of the cervices shown in Figs. 11(c) and 13(c). The depolarization and retardance images in Figs. 10 and 12 do not show any drastic changes as is expected in healthy tissue. Large changes in depolarization and retardance are known to occur in cancerous tissues. ${ }^{44}$

The dashed line box in Fig. 12(a) designates the region that was used to measure the orientation shown in Fig. 13 for both imaging modalities. Circular statistics was applied to the OCT orientation and Mueller matrix decomposed orientation in the same subsections. The selected subsections of the cervices are denoted by the dashed line square boxes in Figs. 11 and 13.

The kurtosis values calculated from the selected cervix region are between 0.74 and 0.78 in comparison to the more ideal values of 0.92 and 0.99 in the nondepolarizing silicon phantom. This is expected due to the strong depolarization effect of biological tissue ${ }^{41,56,57}$ because of its many constituents and the more complex arrangement of collagen in the cervix as opposed to tendon. Another cervix is shown in Fig. 12; as this sample was large, only a section was imaged with OCT, whose field of view is about $\sim 5 \mathrm{~mm}$ while the Mueller Matrix system can measure the full cervix. The dashed line boxes in Figs. 13(a) and 13(c) are the regions selected for the circular statistics calculated in Figs. 13(b) and 13(d), respectively.

A summary of the circumferential fiber alignment of collagen in the middle of the cervix samples is shown in Fig. 14. Eight regions around the OS of the cervix were selected from both the Mueller matrix and the OCT data. The mean orientation and percentage error between the two modalities are plotted for comparison. The mean orientation rotates fully around the cervix and flips sign at the vertical axis (north position). The mean errors between the modalities are $3.26 \%$ and $3.66 \%$, respectively, for the two samples shown. Tissue fixation with paraformaldehyde and paraffin embedding has been shown to cause small increases in depolarization and retardation ${ }^{58-60}$; however, in vivo imaging currently underway which is not shown here, shows very similar results of collagen orientation, hence, we believe that fixation does not alter the ultimate result of this study. We propose that in vivo measurements of collagen
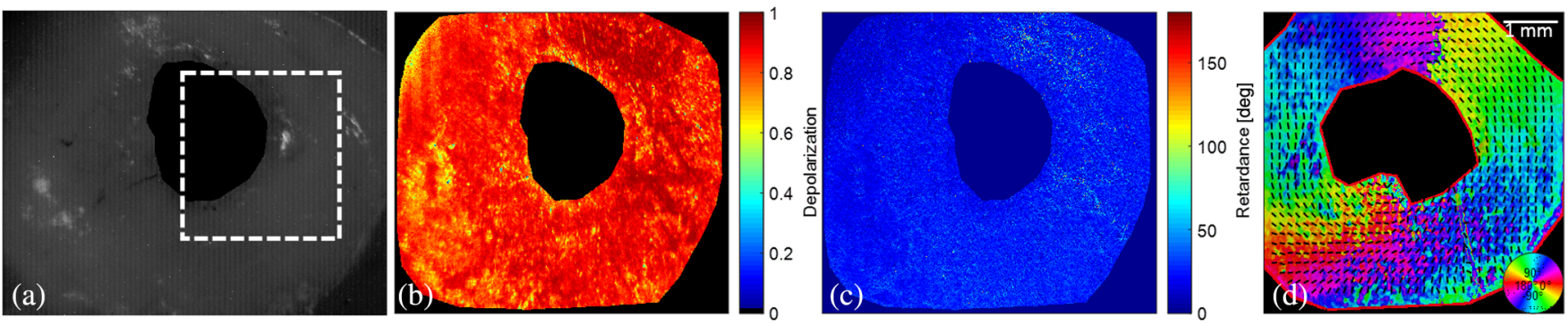

Fig. 12 Paraffin embedded cervix 2 Mueller matrix decomposition: (a) CCD image, (b) Mueller matrix decomposed depolarization, (c) Mueller matrix decomposed retardation, and (d) Mueller matrix decomposed orientation. 

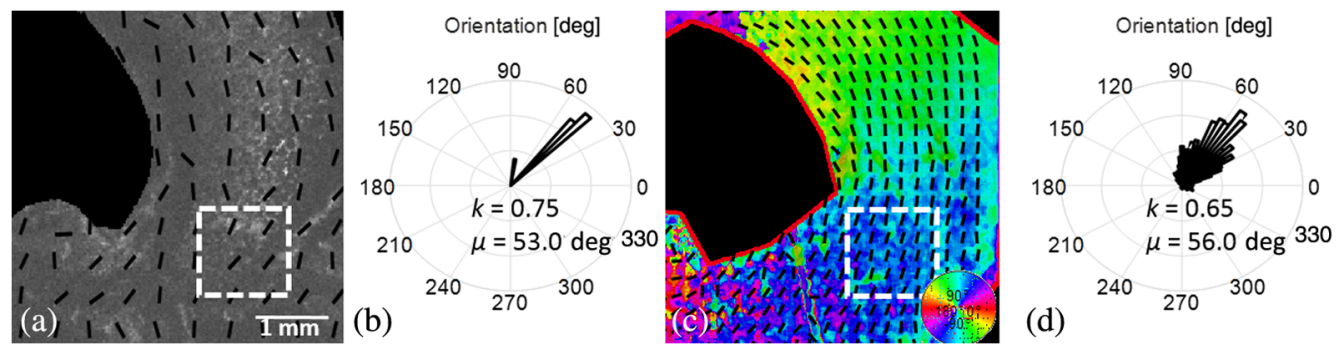

Fig. 13 Paraffin embedded cervix 2 OCT-MMP orientation comparison: (a) OCT c-scan, (c) Mueller matrix decomposed orientation, and (b/d) orientation histogram circular statistics ( $k=$ kurtosis, $\mu=$ mean angle).
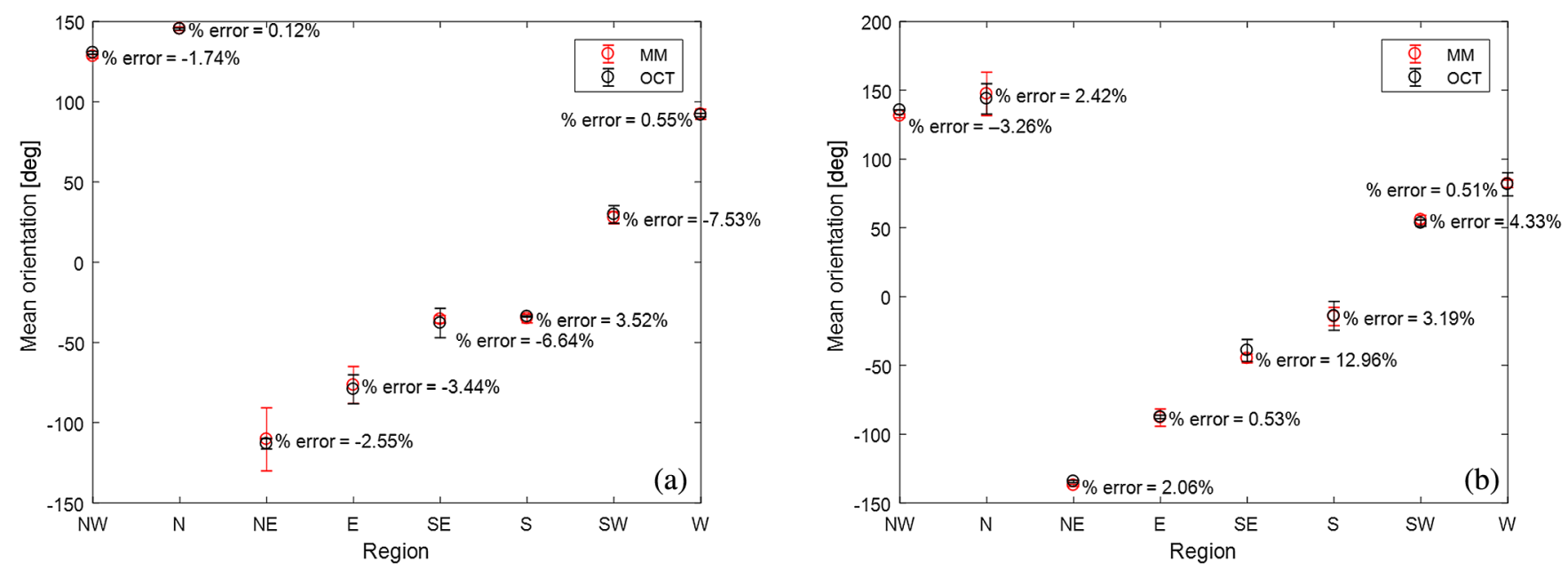

Fig. 14 Summary statistics of cervix orientation between MMP and OCT.

orientation could be used to identify these areas of disruption and lead to diagnosis of abnormal or early collagen disruption in the pregnant cervix.

\section{Conclusion}

Orientation of the optical axis of birefringent material is important in the diagnosis of abnormal conditions in tissues with large amounts of ECM. Tissues that rely on collagen for mechanical strength are often highly anisotropic. ${ }^{61}$ The circumferential orientation of collagen in the cervix is important in maintaining the fetus in the uterus by not allowing passage through the OS and can be measured quickly with one set of images using MMP. To our knowledge, this is the first report that focuses uniquely on polarization-derived collagen orientation in the mammal cervix. Gan et al. ${ }^{40}$ as well as Zhang et al. ${ }^{62}$ have shown that cervical collagen alignment can be measured using OCT and SHG microscopy, respectively. Yet, MMP is more adaptable to cervical imaging studies than those modalities due to its ability to capture the entire cervix at a distance and from outside the vagina. Current modalities for diagnosing preterm labor involve direct contact with the cervix during a pelvic ultrasound ${ }^{63}$ or fibronectin discharge collection. ${ }^{64}$

Depolarization and retardation were also decomposed from the Mueller matrices and showed little variation throughout the different cervical samples. The orientation parameter obtained with MMP was in good agreement with measurements made using OCT. Further studies of collagen orientation in cervices under different conditions are needed to understand if MMP can effectively measure the changes in collagen orientation that should occur when the normal ECM alignment is disrupted by pregnancy or disease. An in vivo study of human cervix is currently underway using a colposcope outfitted with an MM polarimeter of the same design as reported here and will be useful in establishing the diagnostic power of this technique in the determination of risk for preterm labor.

\section{Disclosures}

There is no conflict of interest for the research reported in this article.

\section{References}

1. R. L. Goldenberg et al., "Epidemiology and causes of preterm birth," Lancet 371, 75-84 (2008).

2. K. Myers et al., "The mechanical role of the cervix in pregnancy," J. Biomech. 48(9), 1511-1523 (2015).

3. J. D. Iams and V. Berghella, "Care for women with prior preterm birth," Am. J. Obstet. Gynecol. 203, 89-100 (2010).

4. H. Feltovich, K. Nam, and T. Hall, "Quantitative ultrasound assessment of cervical microstructure," Ultrason. Imaging 32, 131-142 (2010).

5. C. M. Nold et al., "Inflammation promotes a cytokine response and disrupts the cervical epithelial barrier: a possible mechanism of premature cervical remodeling and preterm birth," Am. J. Obstet. Gynecol. 206 , 1-2 (2012).

6. R. A. Word et al., "Dynamics of cervical remodeling during pregnancy and parturition: mechanisms and current concepts," Semin. Reprod. Med. 25, 69-79 (2007). 
7. H. Feltovich, T. J. Hall, and V. Berghella, "Beyond cervical length: emerging technologies for assessing the pregnant cervix," Am. J. Obstet. Gynecol. 207, 345-354 (2012).

8. Y. Zhang et al., "A compact fiber-optic SHG scanning endomicroscope and its application to visualize cervical remodeling during pregnancy," Proc. Natl. Acad. Sci. U.S.A. 109, 12878-12883 (2012).

9. T. Y. Lau et al., "Application of Fourier transform-second-harmonic generation imaging to the rat cervix," J. Microsc. 251, 77-83 (2013).

10. L. M. Reusch et al., "Nonlinear optical microscopy and ultrasound imaging of human cervical structure," J. Biomed. Opt. 18, 031110 (2013).

11. M. L. Akins, K. Luby-Phelps, and M. Mahendroo, "Second harmonic generation imaging as a potential tool for staging pregnancy and predicting preterm birth," J. Biomed. Opt. 15, 026020 (2010).

12. O. Reich and H. Fritsch, "The developmental origin of cervical and vaginal epithelium and their clinical consequences: a systematic review," J. Lower Genital Tract Dis. 18, 358-360 (2014).

13. D. C. Walker et al., "A study of the morphological parameters of cervical squamous epithelium," Physiol. Meas. 24, 121-135 (2003).

14. P. N. Vassilakos and R. Catarino, "Cervical cancer in developing countries," World J. Clin. Oncol. 6(6), 281-290 (2015).

15. P. Mukonoweshuro, A. Oriowolo, and M. Smith, "Audit of the histological definition of cervical transformation zone," J. Clin. Pathol. 58(6), 671 (2005)

16. R. J. Kuon et al., "A novel optical method to assess cervical changes during pregnancy and use to evaluate the effects of progestins on term and preterm labor," Am. J. Obstet. Gynecol. 205, 82.e15-82. e20 (2011).

17. B. Timmons, M. Akins, and M. Mahendroo, "Cervical remodeling during pregnancy and parturition," Trends Endocrinol. Metab. 21, 353-361 (2010).

18. M. House, D. L. Kaplan, and S. Socrate, "Relationships between mechanical properties and extracellular matrix constituents of the cervical stroma during pregnancy," Semin. Perinatol. 33, 300-307 (2009).

19. K. Ito et al., "The change in solubility of type I collagen in human uterine cervix in pregnancy at term," Biomechem. Med. 21, 262-270 (1979).

20. L. Granstrom et al., "Changes in the connective tissue of corpus and cervix uteri during ripening and labour in term pregnancy," $\mathrm{Br}$. $J$. Obstet. Gynaecol. 96, 1198-1202 (1989).

21. K. M. Myers et al., "The mechanical role of the cervix in pregnancy," J. Biomech. 48, 1511-1523 (2015).

22. Biology of the Uterus, R. Wynn, Ed., Springer, New York (1977).

23. M. Iwahashi et al., "Decreased type I collagen expression in human uterine cervix during pregnancy," J. Clin. Endocrinol. Metab. 88, 22312235 (2003).

24. K. M. Myers et al., "Mechanical and biochemical properties of human cervical tissue," Acta Biomater. 4, 104-116 (2008).

25. C. T. Fittkow et al., "Light-induced fluorescence of the human cervix decreases after prostaglandin application for induction of labor at term," Eur. J. Obstet. Gynecol. Reprod. Biol. 123, 62-66 (2005).

26. A. Ito et al., "The role of leukocyte factors on uterine cervical ripening and dilation," Biol. Reprod. 37, 511-517 (1987).

27. J. Ludmir and H. M. Sehdev, "Anatomy and physiology of the uterine cervix," Clin. Obstet. Gynecol. 43, 433-439 (2000).

28. M. L. Akins et al., "Cervical softening during pregnancy: regulated changes in collagen cross-linking and composition of matricellular proteins in the mouse," Biol. Reprod. 84, 1053-1062 (2011).

29. N. M. Zork et al., "A systematic evaluation of collagen cross-links in the human cervix," Am. J. Obstet. Gynecol. 212, 321-321 (2015).

30. A. Blaustein and K. J. Kurman, Blaustein's Pathology of the Female Genital Tract, Springer, New York (2011).

31. K. Myers et al., "Changes in the biochemical constituents and morphologic appearance of the human cervical stroma during pregnancy," Eur. J. Obstet. Gynecol. Reprod. Biol. 144, S82-S89 (2009).

32. L. Shi et al., "Changes in cervical resistance and collagen fluorescence during gestation in rats," J. Perinat. Med. 27, 188-194 (1999).

33. H. Maul et al., "Cervical light-induced fluorescence in humans decreases throughout gestation and before delivery: preliminary observations," Am. J. Obstet. Gynecol. 188, 537-541 (2003).

34. S. Bancelin et al., "Determination of collagen fiber orientation in histological slides using Mueller microscopy and validation by second harmonic generation imaging," Opt. Express 22, 22561-22574 (2014).
35. R. Holt et al., "The molecular mechanisms of cervical ripening differ between term and preterm birth," Endocrinology 152, 1036-1046 (2011).

36. K. M. Myers et al., "A study of the anisotropy and tension/compression behavior of human cervical tissue," J. Biomech. Eng. 132, 021003 (2010).

37. R. M. Aspden, "Collagen organisation in the cervix and its relation to mechanical function," Collagen Relat. Res. 8, 103-112 (1988).

38. M. Fernandez et al., "Investigating the mechanical function of the cervix during pregnancy using finite element models derived from high-resolution 3D MRI," Comput. Meth. Biomech. Biomed. Eng. 19, 404-417 (2016).

39. K. Yoshida et al., "Quantitative evaluation of collagen crosslinks and corresponding tensile mechanical properties in mouse cervical tissue during normal pregnancy," PLoS One 9, e112391 (2014).

40. Y. Gan et al., "Analyzing three-dimensional ultrastructure of human cervical tissue using optical coherence tomography," Biomed. Opt. Express 6, 1090-1108 (2015).

41. J. Chue-Sang et al., "Use of combined polarization-sensitive optical coherence tomography and Mueller matrix imaging for the polarimetric characterization of excised biological tissue," J. Biomed. Opt. 21, 071109 (2016).

42. B. Boulbry, J. C. Ramella-Roman, and T. A. Germer, "Improved method for calibrating a stokes polarimeter," Appl. Opt. 46, 8533-8541 (2007).

43. P. Ghassemi et al., "A new approach for optical assessment of directional anisotropy in turbid media," J. Biophotonics 9, 100-108 (2015).

44. A. Pierangelo et al., "Multispectral Mueller polarimetric imaging detecting residual cancer and cancer regression after neoadjuvant treatment for colorectal carcinomas," J. Biomed. Opt. 18, 046014 (2013).

45. J. Dillet et al., "Size determination by use of two-dimensional Mueller matrices backscattered by optically thick random media," Appl. Opt. 45, 4669-4678 (2006).

46. S. Y. Lu and R. A. Chipman, "Interpretation of Mueller matrices based on polar decomposition," J. Opt. Soc. Am. A 13, 1106-1113 (1996).

47. N. Ghosh, M. F. Wood, and I. A. Vitkin, "Mueller matrix decomposition for extraction of individual polarization parameters from complex turbid media exhibiting multiple scattering, optical activity, and linear birefringence," J. Biomed. Opt. 13, 044036 (2008).

48. N. Ghosh et al., "Mueller matrix decomposition for polarized light assessment of biological tissues," J. Biophotonics 2, 145-156 (2009).

49. M. F. G. Wood et al., "Proof-of-principle demonstration of a Mueller matrix decomposition method for polarized light tissue characterization in vivo," J. Biomed. Opt. 14, 014029 (2009).

50. M. F. Wood, X. Guo, and I. A. Vitkin, "Polarized light propagation in multiply scattering media exhibiting both linear birefringence and optical activity: Monte Carlo model and experimental methodology," J. Biomed. Opt. 12, 014029 (2007).

51. M. Sun et al., "Characterizing the microstructures of biological tissues using Mueller matrix and transformed polarization parameters," Biomed. Opt. Express 5, 4223-4234 (2014).

52. A. Pewsey, "The large-sample joint distribution of key circular statistics," Metrika 60, 25-32 (2004).

53. A. Pewsey, M. Neuhauser, and G. D. Ruxton, Circular Statistics in R, Oxford University Press, Oxford (2015).

54. P. H. Westfall, "Kurtosis as peakedness, 1905-2014. R.I.P," Am. Stat. 68, 191-195 (2014).

55. D. D. Duncan et al., "Absolute blood velocity measured with a modified fundus camera," J. Biomed. Opt. 15, 056014 (2010).

56. S. L. Jacques, J. C. Ramella-Roman, and K. Lee, "Imaging skin pathology with polarized light," J. Biomed. Opt. 7, 329-340 (2002).

57. A. Pierangelo et al., "Polarimetric imaging of uterine cervix: a case study," Opt. Express 21, 14120-14130 (2013).

58. M. F. G. Wood et al., "Effects of formalin fixation on tissue optical polarization properties," Phys. Med. Biol. 56, N115 (2011).

59. C. He et al., "Characterizing microstructures of cancerous tissues using multispectral transformed Mueller matrix polarization parameters," Biomed. Opt. Express 6, 2934-2945 (2015).

60. M. F. G. Wood et al., "Polarized light based birefringence measurements for monitoring myocardial regeneration," Proc. SPIE 7179, 717908 (2009).

61. D.-H. Kim et al., "Matrix nanotopography as a regulator of cell function," J. Cell Biol. 197, 351-360 (2012). 
62. Y. Zhang et al., "A compact fiber-optic SHG scanning endomiscroscope and its application to visualize cervical remodeling during pregnancy," Proc. Natl. Acad. Sci. U.S.A. 109, 12878-12883 (2012).

63. J. D. Iams et al., "The length of the cervix and the risk of spontaneous premature delivery," N. Engl. J. Med. 334, 567-573 (1996).

64. E. F. Foxman and P. Jarolim, "Use of the fetal fibronectin test in decisions to admit to hospital for preterm labor," Clin. Chem. 50, 663-665 (2004).

Joseph Chue-Sang received his BS degree in biomedical engineering from Florida International University (FIU) in 2013. He is a PhD student in biomedical engineering in FIU under Dr. Jessica C. Ramella-Roman and was awarded a SPIE student travel grant to present his work in 2016. He is interested in predicting the risk of preterm birth in pregnant women noninvasively using Mueller matrix polarimetry by characterizing collagen distribution in the cervix.

Yuqiang Bai received his BS degree in physics and his MS degree in optics in China. He completed his $\mathrm{PhD}$ in biomedical engineering from Texas A\&M University in Texas in 2014. He was a visiting postdoctoral associate at FIU, where he developed polarization sensitive optical coherence tomography and two-photon fluorescence microscopy to study anisotropic biological tissues. Now, he is a postdoctoral fellow in school of optometry, University of Alabama at Birmingham.

Mariacarla Gonzalez received her bachelor's of science in chemistry from FIU, Miami in 2014. She is an NSF Bridge to doctorate fellow and an FEF McKnight fellow, and she is currently pursuing graduate studies in biomedical engineering from FIU working under the supervision of Dr. Jessica Ramella-Roman. Her research interests include analysis of collagen modification in heart tissue and the use of polarimetry as a diagnostic tool to observe collagen changes in cervical tissue.

Nola Holness is a clinical assistant professor at FIU. She practices as a certified nurse-midwife at Jackson Memorial Hospital, Miami, Florida. Her program of research is to enhance the reproductive health of adolescent mothers.

Ranu Jung is a fellow of the American Institute of Medical and Biological Engineering and Wallace $\mathrm{H}$. Coulter Eminent Scholar Professor and chair of the Department of Biomedical Engineering at Florida International University. Her research focuses on understanding neural control and developing neurotechnology to restore function after neurotrauma or autonomic disease.

Jessica C. Ramella-Roman is a senior member of SPIE and an associate professor in the Department of Biomedical Engineering at FIU and in the Herbert Wertheim School of Medicine at FIU. Her research focuses on the development of imaging modalities based on spectroscopy and polarization including multimodal applications of nonlinear microscopy.

Biographies for the other authors are not available. 